\title{
MATRICES WITH DEFECT INDEX ONE
}

\author{
Cheng-Chun Chang, Hwa-Long GaU, Ya-Shu Wang, \\ SZU-CHIEH WU AND YA-TING YeH
}

Abstract. In this paper, we give some characterizations of matrices which have defect index one. Recall that an $n$-by- $n$ matrix $A$ is said to be of class $\mathscr{S}_{n}$ (resp., $\mathscr{S}_{n}^{-1}$ ) if its eigenvalues are all in the open unit disc (resp., in the complement of closed unit disc) and $\operatorname{rank}\left(I_{n}-A^{*} A\right)=1$. We show that an $n$-by- $n$ matrix $A$ is of defect index one if and only if $A$ is unitarily equivalent to $U \oplus C$, where $U$ is a $k$-by- $k$ unitary matrix, $0 \leqslant k<n$, and $C$ is either of class $\mathscr{S}_{n-k}$ or of class $\mathscr{S}_{n-k}^{-1}$. We also give a complete characterization of polar decompositions, norms and defect indices of powers of $\mathscr{S}_{n}^{-1}$-matrices. Finally, we consider the numerical ranges of $\mathscr{S}_{n}^{-1}$-matrices and $\mathscr{S}_{n}$-matrices, and give a generalization of a result of Chien and Nakazato on tridiagonal matrices (cf. [3, Theorem 7]).

Mathematics subject classification (2010): 47A12, 15A60.

Keywords and phrases: polar decomposition, defect index, numerical range, $\mathscr{S}_{n}$-matrix, $\mathscr{S}_{n}^{-1}$-matrix.

\section{REFERENCES}

[1] H. Bercovici, Operator Theory and Arithmetic in $H^{\infty}$, Amer. Math. Soc., Providence, RI, 1988.

[2] R. Bhatia, Matrix analysis, Springer, New York, 1997

[3] M. T. Chien, H. NAKaZAto, The numerical range of a tridiagonal operator, J. Math. Anal. Appl. 373 (2011) 297-304.

[4] H.-L. GAU, Numerical ranges of reducible companion matrices, Linear Algebra Appl. 432 (2010) 1310-1321.

[5] H.-L. GaU, P. Y. WU, Numerical range of $S(\phi)$, Linear Multilinear Algebra 45 (1998) 49-73.

[6] H.-L. GAU, P. Y. WU, Lucas' theorem refined, Linear Multilinear Algebra 45 (1999) 359-373.

[7] H.-L. GAU, P. Y. WU, Finite Blaschke products of contractions, Linear Algebra Appl. 368 (2003) 359-370.

[8] H.-L. GaU, P. Y. Wu, Numerical range and Poncelet property, Taiwanese J. Math. 7 (2003) 173-193.

[9] H.-L. GaU, P. Y. WU, Unitary part of a contraction, J. Math. Anal. Appl. 366 (2010) 700-705.

[10] H.-L. GAU, P. Y. WU, Defect indices of powers of a contraction, Linear Algebra Appl. 432 (2010) $2824-2833$.

[11] K. E. Gustafson, D. K. M. RaO, Numerical range, the field of values of linear operators and matrices, Springer, New York, 1997.

[12] R. A. Horn, C. R. Johnson, Topics in matrix analysis, Cambridge University Press, Cambridge, 1991.

[13] C.-K. LI, A note on the unitary part of a contraction, Linear Algebra Appl. 433 (2010) 1533-1535.

[14] B. Mirman, Numerical ranges and Poncelet curves, Linear Algebra Appl. 281 (1998) 59-85.

[15] B. SZ.-NAG Y, C. FoIAŞ, Harmonic Analysis of Operators on Hilbert Space, North Holland, Amsterdam, 1970.

[16] D. SaRAson, Generalized interpolation in $H^{\infty}$, Trans. Amer. Math. Soc. 127 (1967) 179-203.

[17] P. Y. WU, Polar decompositions of $C_{0}(N)$ contractions, Integral Equations Operator Theory, 56 (2006) 559-569.

[18] S.-C. WU, A study on matrices of defect index one, Master Thesis, National Central University, Taiwan, 2008. 